\title{
Heterodyne speckle velocimetry of Poiseuille flow
}

\author{
M. D. Alaimo and M. A. C. Potenza \\ Dipartimento di Fisica, Università di Milano, Via Celoria 16, Milano I-20133, Italy \\ D. Magatti and F. Ferri ${ }^{\text {a) }}$ \\ Dipartimento di Fisica e Matematica, Università dell'Insubria, Via Valleggio 11, Como I-22100, Italy
}

(Received 8 May 2007; accepted 14 August 2007; published online 12 October 2007)

\begin{abstract}
We review the technique named heterodyne speckle velocimetry and present two applications for testing the method with a fluid moving under the conditions of Poiseuille flow. The fluid was seeded with small tracking particles (diameter $300 \mathrm{~nm}$ ) and fluxed between the two parallel planes of a cell with constant or variable cross section. In the first case the velocity distribution was constant along the direction parallel to the planes and was in excellent agreement with the expected Poiseuille profile along the orthogonal direction. In the second case, where velocity gradients were present also along the planes, the technique was able to reconstruct both the orthogonal Poiseuille profile and the in-plane two dimensional mapping of the velocity vectors, with the possibility of measuring the fluid flux within an accuracy of 1\%. (C) 2007 American Institute of Physics. [DOI: 10.1063/1.2786905]
\end{abstract}

\section{INTRODUCTION}

Experiments in modern fluid dynamics require fast and possibly simple measurements of fluid flows in three dimensional (3D) domains. This task is quite challenging and is usually accomplished by seeding the fluid with small tracking particles, illuminating the sample with some light, and detecting the scattered light.

There are many techniques devoted to this aim, ${ }^{1}$ the most popular ones being particle imaging velocimetry (PIV), ${ }^{2}$ laser speckle velocimetry (LSV), ${ }^{3}$ laser doppler velocimetry (LDV), ${ }^{4}$ holographic PIV (HPIV), ${ }^{5}$ and all the various implementations which have been proposed over the years. $^{6-8}$

In their simplest realizations, PIV and LSV work by illuminating the seeded fluid with a thin sheet of light and imaging the particles along a perpendicular direction. Depending on the numerical aperture (NA) of the collection optics as well as on the tracer concentration, the particles may be resolved (PIV) or not (LSV). In any case, by taking the spatial cross correlation between two images acquired at two closely spaced times, one can determine the local tracer displacements and consequently recover the two dimensional (2D) in-plane velocity mapping of the fluid. PIV can also be implemented by using simultaneously two sheets of light at a given angle: in this way 3D stereo reconstruction of the fluid velocities can be carried out, but the apparatus and the overall procedure become rather complex.

LDV works by illuminating the seeded fluid with two laser beams, which produce a set of straight fringes inside the sample. Particles crossing the fringes produce a signal whose periodicity carries information about the fluid velocity component perpendicular to the fringes. Thus LDV appears to be rather simpler than PIV or LSV, but the information is necessarily averaged over the illuminated region and is restricted to only one component of the velocity vector. Imple-

\footnotetext{
a) Author to whom correspondence should be addressed. Electronic mail: fabio.ferri@uninsubria.it
}

mentations for the measurement of all the three velocity components can be done, but the full 3D mapping of the fluid velocities is hard to be recovered.

A much powerful technique is represented by HPIV, which, in principle, allows the complete recovery of the $3 \mathrm{D}$ positions of all the tracers with a high spatial (diffraction limited) resolution. However, this method presents various nonsecondary drawbacks, related to its holographic nature: the setup may be rather complex, the data reduction is usually not straightforward and it can be hardly carried out in real time, and, finally, the scattering signal of each single particle has to be strong enough to beat the noise of the system. The last requirement is related to the system sensitivity and, ultimately, determines the minimum size of the tracers (microns).

In a recent article ${ }^{9}$ we have proposed a velocimetry technique called heterodyne speckle velocimetry (HSV), which provides instantaneous $2 \mathrm{D}$ velocity mappings and velocity distributions of a fluid moving in a plane. The method, developed in the context of the topic called near field scattering, ${ }^{10-13}$ adopts a setup similar to the one used in the in-line HPIV, ${ }^{14}$ in which the strong transmitted beam passing through the seeded fluid interferes with the weak fields scattered by the tracking particles. If the observation plane is close enough to the sample, the heterodyne speckles originated from this interference process move rigidly with the tracers, and a 2D mapping of fluid velocities can be recovered by cross correlating two images taken at a close temporal distance. The method is clearly less informative than (stereo) PIV or HPIV, but offers several advantages deriving from the large statistical information contained in the speckle field: it is fairly sensitive because it profits from the contributions deriving from the whole sample thickness; it works well with particles smaller than the wavelength of light whose concentration can also be relatively high; it uses a rather simple setup, in which the alignment requirements are rather relaxed and the rejection of stray light is highly efficient; the data analysis is fairly simple and fast, allowing for 


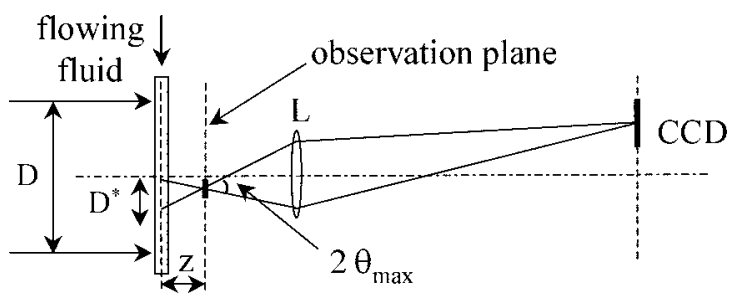

FIG. 1. Schematic diagram of the experimental setup. A collimated laser beam of diameter $D$ illuminates the fluid moving inside a square cell. The interference between the transmitted and the scattered light is recorded at a close distance $z$ from the cell by using the lens $L$. The maximum acceptance angle $2 \theta_{\max }$ together with the distance $z$ determines the size $D^{*}$ of the region from which the light reaching the CCD sensor is actually collected.

a real time data processing; and finally, the heterodyne condition guarantees the linearity of the method with respect to the number of particles, and this is a key ingredient when velocity distributions are to be recovered.

This article presents two applications of the HSV technique, aimed to show how the motion of a fluid moving under the conditions of Poiseuille flow can be quantitatively characterized. The article is organized as follows: Sec. II is devoted to review and summarize the main feature of the HSV technique; Sec. III describes two simulations that show the conditions under which the technique can be profitably used; the two experiments describing Poiseuille flows in a square and funnel-shaped cell are described in Secs. IV and $\mathrm{V}$, respectively; and conclusions are reported in Sec. VI.

\section{THE HETERODYNE SPECKLE VELOCIMETRY (HSV) TECHNIQUE}

The HSV technique and its optical setup (Fig. 1) are both very simple: a large collimated laser beam (diameter $D$, wavelength $\lambda$ ) is sent onto a square cell containing small tracking particles (diameter $d$ ) moving with the fluid. The interference between the intense transmitted beam and the weak wave fronts scattered by the particles generates a speckle field, whose intensity distribution can be detected at a close distance $z$ from the cell by using a charge coupled device (CCD) camera and an optical microscope objective. If the particles are sufficiently small $(d \leqslant \lambda)$ the NA of the microscope determines the maximum scattering angle $\left(\theta_{\max }\right.$ $\sim$ NA) collected by the optics, and in turn, the size $\delta$ of the (subjective) speckles, namely, $\delta \sim \lambda /\left(2 \theta_{\max }\right)$. If the distance $z$ of the observation plane is sufficiently small, the transversal region from which the scattered light is collected, $D^{*}$ $\sim 2 z \theta_{\text {max }}$, is smaller than the beam size $D$. When this condition is strongly fulfilled $\left(D^{*} \ll D\right)$, we are in the so called near field condition, and the speckle pattern formed around any given point $\mathbf{r}$ of the observation plane is determined only by those particles lying within the region $D^{*}$ centered on the back projection of $\mathbf{r}$ over the scattering cell. Thus, if the sample moves transversally to the optical axis, the speckles move accordingly, and by comparing two snapshots of these speckle patterns at known times, one can recover the displacements of the fluid during the time elapsed.

Let us indicate with $e_{S}(\mathbf{r}, t)$ and $e_{0}$ the scattered and the transmitted fields, respectively. Under the assumption $e_{S}$ $\ll e_{0}$, we can write the intensity distribution $f(\mathbf{r}, t)$ as

$$
f(\mathbf{r}, t)=\left|e_{0}\right|^{2}+2 \operatorname{Re}\left\{e_{0}^{*} e_{S}(\mathbf{r}, t)\right\},
$$

in which the term $\left|e_{S}\right|^{2}$ has been neglected. In Eq. (1) $\left|e_{0}\right|^{2}$ represents the strong static transmitted intensity while the last term, being related to the random time-dependent particle positions, is a small stochastic zero-average fluctuating term which is customarily called heterodyne signal. By taking the difference between two intensity distributions detected at a time distance $\tau$, we can determine the differential heterodyne signal

$$
\begin{aligned}
\delta f(\mathbf{r}, t, \tau) & =f(\mathbf{r}, t+\tau)-f(\mathbf{r}, t) \\
& =2 \operatorname{Re}\left\{e_{0}^{*}\left[e_{s}(\mathbf{r}, t+\tau)-e_{s}(\mathbf{r}, t)\right]\right\},
\end{aligned}
$$

which is clearly independent of $\left|e_{0}\right|^{2}$. By computing the autocorrelation function of $\delta f(\mathbf{r}, t, \tau)$, we get

$$
g_{\tau}(\mathbf{x})=4\left|e_{0}\right|^{2} \operatorname{Re}\{2 G(\mathbf{x}, 0)-G(\mathbf{x}, \tau)-G(-\mathbf{x}, \tau)\},
$$

in which $G(\mathbf{x}, \tau)$ is the mutual coherence function of the scattered field defined as ${ }^{15}$

$$
G(\mathbf{x}, \tau)=\left\langle e_{S}(\mathbf{r}, t) e_{S}^{*}(\mathbf{r}+\mathbf{x}, t+\tau)\right\rangle,
$$

where $\langle\cdot\rangle$. denotes ensemble average.

The first term in Eq. (3), $G(\mathbf{x}, 0)$, is positive and represents the spatial autocorrelation function of the (frozen) scattered field. As known, ${ }^{15,9} G(\mathbf{x}, 0)$ is linear in the number of particles and, therefore, can be written as

$$
G(\mathbf{x}, 0)=N^{*}\left\langle\left|a_{S}\right|^{2}\right\rangle h(\mathbf{x}),
$$

in which $N^{*}$ is the number of particles lying inside the region $D^{*}$ and $\left\langle\left|a_{s}\right|^{2}\right\rangle$ is the average intensity scattered by each particle and falling onto the sensor. The function $h(\mathbf{x})$ is the normalized $[h(0)=1]$ autocorrelation function of the speckle field and describes the shape of the speckles. In particular, its width determines the average speckle size $\delta$. The last two terms appearing in Eq. (3) are negative cross-correlation terms, which depend on particle motion. Note that since the latter terms are always present together, $g_{\tau}(\mathbf{x})$ is symmetrical with respect to $\mathbf{x}=0$, and carries information only about the direction and modulus of the fluid velocity, but not about its sign.

When all the particles move with the same average velocity $\langle\mathbf{v}\rangle$ transverse to the optical axis, the scattered field at the observation plane in $\mathbf{r}+\Delta \mathbf{r}$ at the time $t+\tau$ is simply a "shifted version" of the field in $\mathbf{r}$ at the time $t$. Under this hypothesis, it is straightforward to show that ${ }^{9}$

$$
G(\mathbf{x}, \tau)=G(\mathbf{x}-\Delta \mathbf{r}, 0),
$$

in which $\Delta \mathbf{r}=\langle\mathbf{v}\rangle \tau$. Thus, the cross-correlation function $G(\mathbf{x}, \tau)$ is simply a shifted version of the autocorrelation function $G(\mathbf{x}, 0)$ and, thanks to Eq. (5), it is also linear in the number of particles. As a consequence, $G(\mathbf{x}, \tau)$ is proportional to the number of particles that have been displaced by $\mathbf{x}=\Delta \mathbf{r}$ during the time $\tau$, and, if particle motion is characterized by a distribution of displacements $n[\Delta \mathbf{r}(\tau)]$, the resulting cross-correlation function is given by the convolution integral 


$$
G(\mathbf{x}, \tau) \sim \int n(\Delta \mathbf{r}) h(\mathbf{x}-\Delta \mathbf{r}) d \Delta \mathbf{r},
$$

in which the convolving function is the normalized autocorrelation function $h(\mathbf{x})$ defined in Eq. (5). The spatial resolution of the method is therefore set by the width of $h(\mathbf{x})$, i.e., by the average speckle size $\delta$, and two close displacements $\Delta \mathbf{r}$ apart can be resolved only if $\Delta \mathbf{r} \geqslant \delta$.

The average speckle size $\delta$ also determines the smallest particle diameter suitable for the technique: if particle displacements are to be detected with an accuracy of $\delta$, their rms distance $\Delta s$ traveled by diffusion during the time $\tau$, along any given direction $(\Delta s=\sqrt{2 D \tau}, D$ being the diffusion coefficient), has to be smaller than $\delta$. Thus, imposing $\Delta s$ $<\delta$, we find that the particle diameter $d$ must be larger than

$$
d>\frac{2 k_{B} T}{3 \pi \eta} \frac{\tau}{\delta^{2}} \sim 0.88\left(\mu \mathrm{m}^{3} / s\right) \frac{\tau}{\delta^{2}},
$$

in which we have used the classical Stokes-Einstein relation, ${ }^{16}$ with $k_{B}$ being the Boltzmann constant, $T$ the absolute temperature, and $\eta$ the dynamical viscosity of the fluid. The numerical factor appearing in the right-hand side (rhs) of Eq. (8) helps to determine quantitatively the minimum particle diameter usable under standard conditions $(T$ $\left.=300 \mathrm{~K}, \quad \eta=0.01 \mathrm{~g} \mathrm{~cm}^{-1} \mathrm{~s}^{-1}\right)$. In our case $(\tau=0.1 \mathrm{~s}, \delta$ $\sim 4.5 \mu \mathrm{m}$, see below) we have $d>4.3 \mathrm{~nm}$, which is by far smaller than the particle diameter used in the experiment $(d=300 \mathrm{~nm})$.

\section{SIMULATIONS}

In this section we present two simulations aimed to show how HSV works when a fluid seeded with small particles moves orthogonal to the optical axis.

The first simulation describes what happens when the fluid is uniformly displaced by the vector $\Delta \mathbf{r}$, or equivalently, when all the particles move with the same average velocity $\langle\mathbf{v}\rangle$ over the time $\tau$, so that $\Delta \mathbf{r}=\langle\mathbf{v}\rangle \tau$. The simulation is carried out as follows: we suppose to have $N_{\text {part }}$ par-
TABLE I. Optical parameters used for simulation Nos. 1 and 2 described in Sec. III.

\begin{tabular}{lll}
\hline \hline Laser wavelength & $\lambda$ & $0.6328 \mu \mathrm{m}$ \\
Cell diameter & $D$ & $2 \mathrm{~mm}$ \\
Cell thickness & $h$ & $1.0 \mathrm{~mm}$ \\
Cell-sensor distance & $z$ & $2 \mathrm{~mm}$ \\
Collecting optics aperture & $\mathrm{NA}$ & 0.1 \\
CCD effective side & $L$ & $854 \mu \mathrm{m}$ \\
CCD number of pixels & $N_{\text {pxl }} \times N_{\text {pxl }}$ & $512 \times 512$ \\
Particle diameter & $d$ & $300 \mathrm{~nm}$ \\
Number of particles & $N_{\text {part }}$ & $10^{4}$ \\
Scattering approximation & & Rayleigh-Gans \\
$e_{s} / e_{0}($ rms $)$ & & $10^{-4}$ \\
Displacement (sim. No. 1) & $\Delta \mathbf{r}$ & $100 \mu \mathrm{m}$ \\
Displacement (sim. No. 2) & $\Delta \mathbf{r} \pm\left\langle\delta \mathbf{r}^{2}\right\rangle^{1 / 2}$ & $100 \pm 5 \mu \mathrm{m}$ \\
\hline \hline
\end{tabular}

ticles of diameter $d$, randomly distributed inside a circular scattering cell of diameter $D$ and thickness $h$. A CCD sensor made of $N_{\text {pxl }} \times N_{\text {pxl }}$ pixels arranged onto a square grid is placed at a distance $z$ from the center of the cell. A uniform laser beam of wavelength $\lambda$ and field amplitude $e_{0}$ is shined onto the sample and, for each particle, the (complex) scattered field distribution in correspondence of the sensor plane is computed by using the Rayleigh-Gans ${ }^{17}$ approximation. The procedure is repeated for every particle, and the overall scattered field $e_{s}$ is obtained by summing up all the contributions. Thus, the scattered intensity $f_{1}(\mathbf{r})$ falling onto the sensor is computed by letting $e_{s}$ interfere with $e_{0}$ (after propagation). We then displace rigidly all the particles by the vector $\Delta \mathbf{r}$, orthogonal to the optical axis, repeat the procedure, and compute $f_{2}(\mathbf{r})$. The differential heterodyne speckle field can be finally recovered as $\delta f_{12}(\mathbf{r})=f_{2}(\mathbf{r})-f_{1}(\mathbf{r})$.

All the optical parameters of the simulation were chosen so to closely reproduce the conditions of the setup of Fig. 1, in which the light is collected by using a microscope objective $(4 \times, N A=0.1)$ and imaged onto a CDD sensor with physical squared pixels of size $6.67 \mu \mathrm{m}$. A summary of all the used parameters is reported in Table I.

The differential heterodyne speckle field $\delta f_{12}(\mathbf{r})$ and the
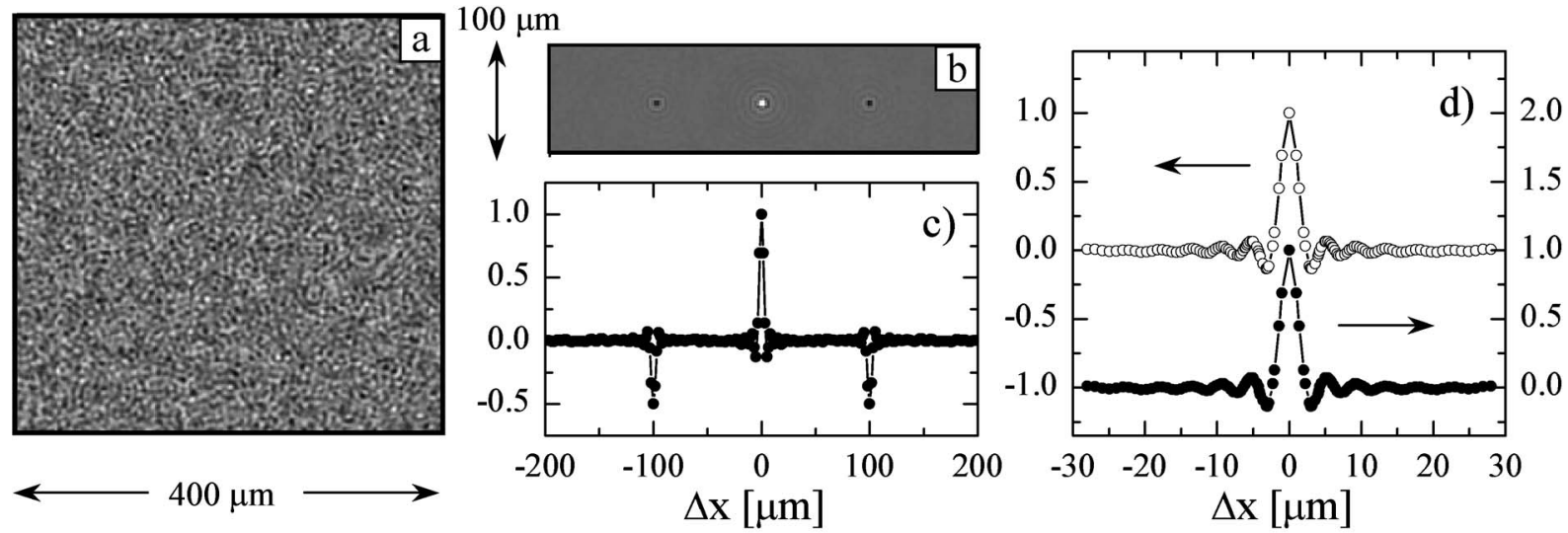

FIG. 2. Results of simulation No. 1 corresponding to a horizontal rigid displacement $\Delta \mathbf{r}=100 \mu \mathrm{m}$ of all the tracking particles: (a) differential heterodyne signal $\delta f_{12}(\mathbf{r})$, appearing as uniform speckle field where particle displacement is completely hidden; (b) autocorrelation function $g_{12}(\mathbf{x})$ showing one central positive peak corresponding to $G(\mathbf{x}, 0)$ and two lateral negative symmetric peaks corresponding to $G(\mathbf{x}, \tau)$; (c) horizontal section of $g_{12}(\mathbf{x})$ passing through the centers of the three peaks; and (d) comparison between radial profiles of $G(\mathbf{x}, 0)$ (open circles, left scale) and $G(\mathbf{x}, \tau)$ (solid circle, right scale). For the sake of clarity, the $G(\mathbf{x}, \tau)$ peak has been multiplied by 2 and inverted. As expected [see Eq. (6)], the two peaks appear to be identical, with a FWHM speckle size $\delta \sim 4.5 \mu \mathrm{m}$. 

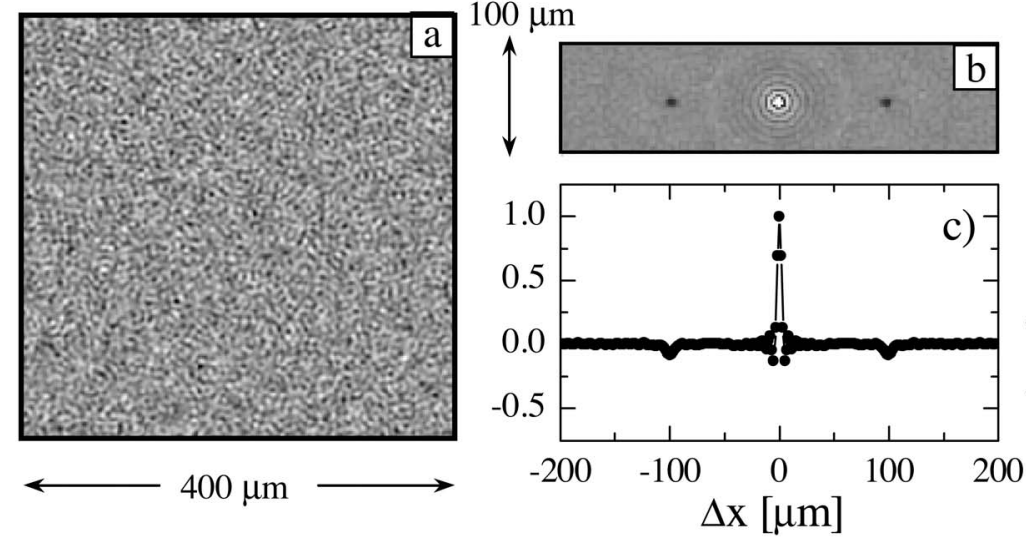

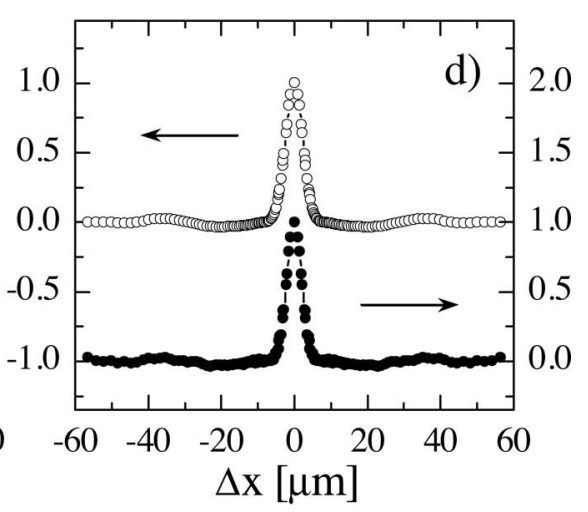

FIG. 3. Results of simulation No. 2 corresponding to a particle horizontal displacement $\Delta \mathbf{r}=100 \pm 5 \mu \mathrm{m}$ : (a) differential heterodyne signal $\delta f_{12}(\mathbf{r})$, statistically identical to Fig. 2(a); (b) autocorrelation function $g_{12}(\mathbf{x})$ characterized by a central peak $[G(\mathbf{x}, 0)]$ identical to the one of Fig. 2(b), and by two lateral peaks $[G(\mathbf{x}, \tau)]$ much dimmer and wider than the ones of Fig. 2(b); (c) horizontal section of $g_{12}(\mathbf{x})$ passing through the centers of the three peaks; (d) comparison between the radial profiles of $G(\mathbf{x}, \tau)$ describing the displacement distributions of the particles (open circles, left scale) and the expected theoretical distribution (solid circles, right scale). For the sake of clarity, the $G(\mathbf{x}, \tau)$ peak has been multiplied by 2 and inverted.

corresponding correlation function $g_{12}(\mathbf{x})$ are shown in Figs. $2(\mathrm{a})-2(\mathrm{~d})$, respectively. As one can notice, $\delta f_{12}(\mathbf{r})$ is a uniform speckle field where particle displacement is completely hidden. Conversely, $g_{12}(\mathbf{x})$ does carry this information in the double twin dark negative spots [Fig. 2(b)], which are located at the same distance $\Delta \mathbf{r}=100 \mu \mathrm{m}$ from the central white positive spot, as shown by the horizontal section of $g_{12}(\mathbf{x})$ [Fig. 2(c)]. One can also notice that the amplitude of the central positive peak associated with the autocorrelation $G(\mathbf{x}, 0)$ is twice the amplitude of two negative lateral peaks associated with the cross correlation $G(\mathbf{x}, \tau)$, consistent with Eq. (3). However, Fig. 2(c) is a rather poor sampling of Fig. 2(b), and a much better analysis can be carried out by taking the radial profiles of the positive and negative spots, each one around its own center. The result is reported in Fig. 2(d), in which the profile relative to $G(\mathbf{x}, \tau)$ (solid circles, right scale) has been doubled, inverted, and compared with the $G(\mathbf{x}, 0)$ profile (open circles, left scale). Figure 2(d) shows that shapes of the two peaks are identical, consistent with the expectation that a single particle displacement $\Delta \mathbf{r}$ appears as convolved with the speckle field autocorrelation function $G(\mathbf{x}, 0)$, i.e., the white central spot. Finally from Fig. 2(d), we can estimate the size of the speckles as the full width at half maximum (FWHM) of $G(\mathbf{x}, 0)$, obtaining $\delta \sim 4.5 \mu \mathrm{m}$.

In the second simulation, we repeated the procedure described above, i.e., displacing all the particles by the same vector $\Delta \mathbf{r}$, but also letting them diffuse transversally according to a Gaussian distribution characterized by a root mean square displacement $\mathbf{a}=\left\langle\delta \mathbf{r}^{2}\right\rangle^{1 / 2}=5 \mu \mathrm{m}$. All the other parameters were kept identical to the ones used in the first simulation and reported in Table I. Figure 3(a) reports the differential heterodyne signal $\delta f_{12}(\mathbf{r})$, which appears to be statistically undistinguishable from the one of Fig. 2(a), carrying no evident information on particle motion. Figures 3(b) and 3(c) show that the correlation function $g_{12}(\mathbf{x})$ exhibits the same white central spot of Fig. 2(b), but the lateral spots are much dimmer and somewhat wider, though centered around the same displacement vector $\Delta \mathbf{r}=100 \mu \mathrm{m}$. Finally, Fig. 3(d) compares the radial profiles of $G(\mathbf{x}, \tau)$ describing the displacement distributions of the particles (open circles) with the expected theoretical distribution (solid circles). The latter one has been determined by convolving (2D) the Gaussian distribution used for generating the particle displacements with the autocorrelation function $G(\mathbf{x}, 0)$. The matching of the two curves is remarkably good, showing that the method is able to accurately recover particle displacement (or velocity) distributions.

\section{EXPERIMENTAL STUDY OF THE POISEUILLE FLOW}

Let us consider a fluid confined between two parallel vertical plates distant $h$ and centered in $z=0$. If a pressure gradient $(d p / d y)$ is present along the $y$ axis, under the hypothesis of laminar flow, the velocity profile along $z$ is given by ${ }^{16}$

$$
v(z)=\frac{1}{2 \eta} \frac{d p}{d y}\left[\frac{h^{2}}{4}-z^{2}\right],
$$

where $\eta$ is the dynamical viscosity. From Eq. (9) it can be easily worked out that the probability density function for the velocity distribution is

$$
P(v)=\frac{1}{2 v_{0} \sqrt{1-v / v_{0}}},
$$

where $v_{0}$ is the maximum velocity located in the middle of the cell $(z=0)$, given by $v_{0}=(d p / d y) h^{2} / 8 \eta$. As it can be seen, the only free parameter appearing in the function $P(v)$ is the maximum velocity $v_{0}$, which is ultimately determined by the experimental conditions and related to the average velocity by

$$
\langle v\rangle=(2 / 3) v_{0}
$$

as it can be easily demonstrated by integrating Eq. (10).

To implement a system exhibiting a Poiseuille flow, we assembled a homemade cell (1.0 mm thick, $15 \mathrm{~mm}$ wide), made of two optical windows separated by a compressed and straightened O-ring (see Fig. 4). The fluid was a water sus- 


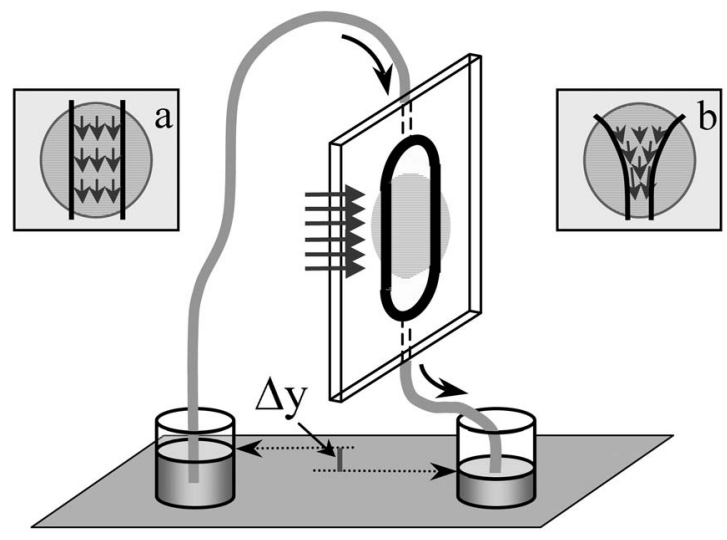

FIG. 4. Schematic picture of the cell used for studying the Poiseuille flow described in Secs. IV and V. The two vertical optical windows are separated by an O-ring, compressed, and straightened so to make a cell with a constant (inset a) and a variable (inset b) cross section. The flow is controlled by the difference in the liquid levels of the two jars. The upper left and right insets show the shapes of the cell used for the experiments described in Sec. IV and $\mathrm{V}$, respectively.

pension of latex particles (diameter $300 \mathrm{~nm}$ ) and a laminar flow (Reynolds number $\mathrm{Re} \sim 1$ ) was induced by the difference $\Delta y$ in the levels of the fluids in the two jars. The vials' diameter was much larger than the cell cross section, so that, in spite of the fact that the pressure gradient (and consequently $v_{0}$ ) progressively reduces, it could be considered constant during the time interval $\tau$. The cell was illuminated with a $\mathrm{He}-\mathrm{Ne}$ laser beam $(\lambda=0.6328 \mu \mathrm{m}, D=20 \mathrm{~mm}$ at $\left.1 / e^{2}\right)$, the collection optics was a $4 \times$ microscope objective $(\mathrm{NA}=0.1)$, and the observation plane was set at a distance $z=2 \mathrm{~mm}$. The frames were acquired at a rate of $10 \mathrm{~Hz}$ and the overall measuring time was $T \sim 30 \mathrm{~s}$. Figure 5(a) shows a typical differential heterodyne signal $\delta f(\mathbf{r}, t, \tau)$ obtained for $\tau=0.1 \mathrm{~s}$, in which no fluid motion is evident. Conversely, the fluid flow becomes evident in the spatial 2D-correlation function $g_{\tau}(\mathbf{x})$ (obtained by averaging ten frames), as shown in Fig. 5(b). This figure shows, besides the central positive spot associated with the speckle autocorrelation function, two symmetrical negative elongated streams that start from the center and become more and more intense, up to a maximum distance, beyond which they disappear. This feature indicates the presence of a velocity distribution inside that region, as expected for a Poiseuille flow. From Fig. 5(b) we estimated that the maximum fluid velocity was $v_{0}$ $\sim 817 \mu \mathrm{m} / \mathrm{s}$, which corresponds to an average velocity $\langle v\rangle$ $=(2 / 3) v_{0} \sim 545 \mu \mathrm{m} / \mathrm{s}$. Thus, taking into account that the thickness and the width of the cell were 1 and $15 \mathrm{~mm}$, respectively, the fluid flux $(\langle v\rangle$ times cell cross section) was $8.18 \mathrm{~mm}^{3} / \mathrm{s}$.

The velocity probability density distribution $P(v)$ was recovered by extracting, for each $g_{\tau}(\mathbf{x})$, the onedimensional (1D) section along the flow direction so that $P(v)=g_{\tau}(x=0, y / \tau)$. Then, in order to take into account the time dependence of the maximum velocity $v_{0}$, for each section we determined $v_{0}$, multiplied $P(v)$ times $v_{0}$, and rescaled the velocities by $v_{0}$. In this way we obtained a timeindependent master curve, which was averaged over the entire measuring time ( $\sim 300$ frames). The result is shown in Fig. 6 (circles), in which we report, as a function of $\alpha$ $=v / v_{0}$, the flow velocity rescaled density distribution $v_{0} P(v)$. The data corresponding to the autocorrelation peak $(\alpha \ll 1)$ have been omitted. The typical noise level for this kind of measurements is indicated by the behavior of fluctuating data around zero (dots), which were obtained by taking a (averaged) section of $g_{\tau}(\mathbf{x})$, not passing for the flow direction. As it is evident from Fig. 6, the maximum velocity turns out to be also the most probable one, while the velocities in the limit $\alpha=0$ are also populated. Both these are features expected for a viscous fluid moving between two parallel walls, that is, faster in the middle and stationary at the boundaries.

A comparison between the data shown in Fig. 6 and the theoretical prediction of Eq. (10) was carried out by taking
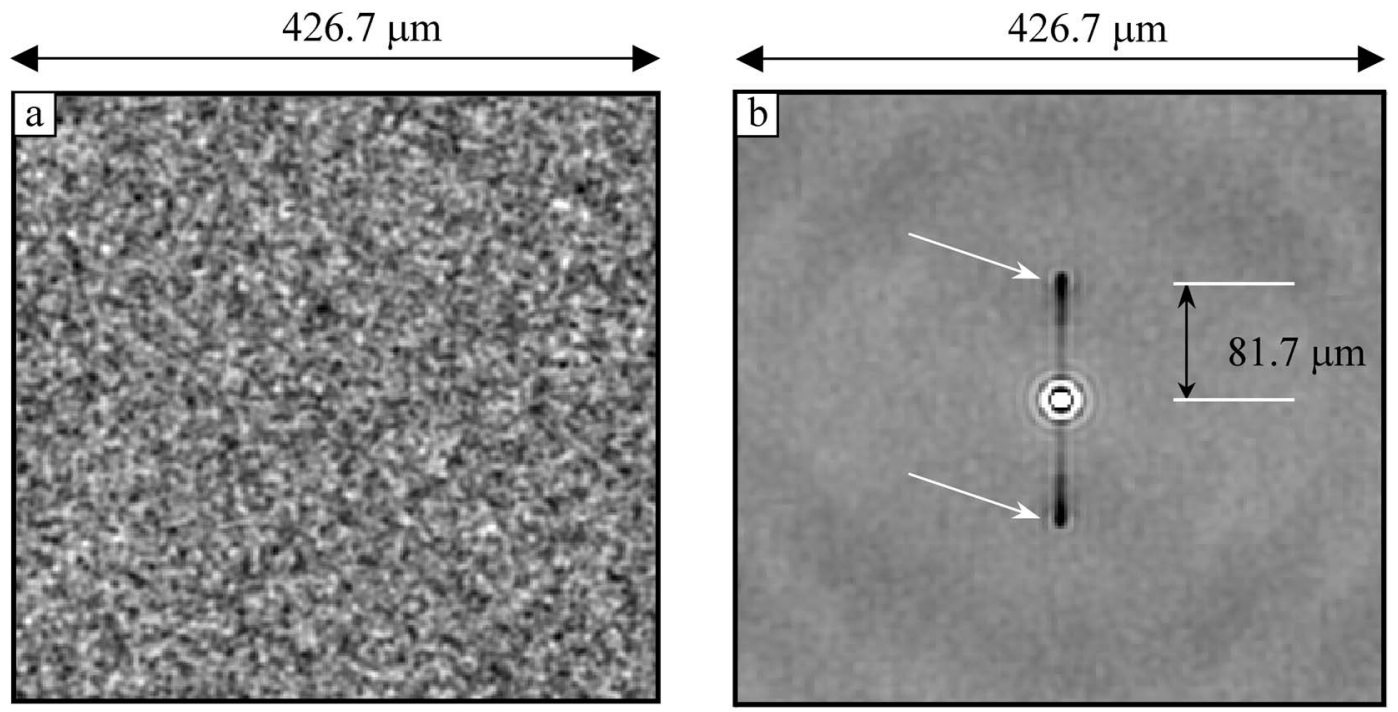

FIG. 5. Experimental heterodyne signal $\delta f(\mathbf{r}, t, \tau)$ (a) and the corresponding autocorrelation function $g_{\tau}(\mathbf{x})$ (b) obtained for $\tau=0.1 \mathrm{~s}$, describing the Poiseuille flow inside the cell of Fig. 4 (inset a). While in $\delta f(\mathbf{r}, t, \tau)$ no fluid motion is evident, $g_{\tau}(\mathbf{x})$ carries this information. Note that the white central peak associated with $G(\mathbf{x}, 0)$ is quite similar to the ones shown in the simulations [Figs. 2(b) and 3(b)]; the negative contributions associated with $G(\mathbf{x}, \tau)$ are completely different, appearing as elongated streams that die beyond a maximum distance indicated by the white arrows. 


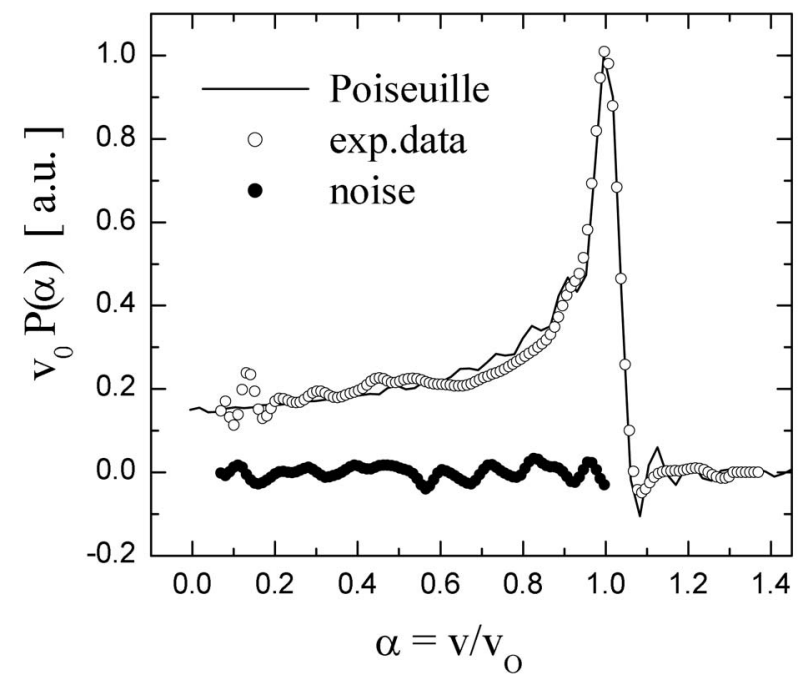

FIG. 6. Master curve describing the velocity distribution of the Poiseuille flow occurring inside the cell of Fig. 4 (inset a). The experimental data (circles) are compared with the expected Poiseuille profile (solid curve) after taking into account the finite spatial resolution of the optical setup. Typical noise of the system is shown by the data fluctuating around zero (dots).

into account the convolution integral given by Eq. (7). Following the same procedure described in Sec. III, we computed numerically the 2D-autocorrelation function of the setup, and then convolved this function with the theoretical probability density distribution $P(v)$ given by Eq. (10).

In this way we obtained a 2D image whose section, reported in Fig. 6 as a continuous line, exhibits the typical oscillations that characterize the autocorrelation function. The matching between this curve and the experimental data is excellent, showing the effectiveness of the technique for recovering velocity density distributions.

\section{2D MAPPING OF THE POISEUILLE FLOW}

The second important feature of the HSV technique comes from the almost local character of Eq. (5). Indeed the displacement of the speckles at a given position $\mathbf{r}$ of the sensor is determined only by the motion of the $N^{*}$ scatterers inside the portion $D^{*}$ of the illuminated sample (see Fig. 1). Thus, if we locate the (effective) sensor plane very close or inside the sample $(z=0)$, it is possible to establish almost a one-to-one mapping between pixel position and transverse position inside the cell. In this way it is possible to realize a mapping of the fluid velocities in the plane transverse to the optical axis and study velocity gradients present in the fluid.

To show this feature, we modified the cell of Fig. 2 by moving the O-ring so to taper the cell width similar to the shape of a funnel (see inset of Fig. 4). The rest of the setup was identical to the one described in Sec. IV, but with $z=0$. An example of the signal $\delta f(\mathbf{r}, t, \tau)$ detected for $\tau=0.1 \mathrm{~s}$ is reported in Fig. 7(a), which shows a quite uniform speckle field inside the entire funnel profile. As for the case of Fig. 5(a), no evidence of fluid motion is appreciable. The analysis was carried out by dividing the entire frame of Fig. 7(a) into $16 \times 16$ patches of $107 \times 107 \mu \mathrm{m}^{2}$, and, for each patch, the autocorrelation function $g_{\tau}(\mathbf{x})$ was carried out. The result is shown in Fig. 7(b), in which the Poiseuille streams are fairly evident (see insets). As expected, the velocity is higher in the middle of the channel than at the boundaries, and increases as the funnel neck gets narrower.

The quantitative analysis of Fig. 7(b) is shown in Fig. $7(\mathrm{c})$, in which each dotted segment indicates modulus and direction of the fluid velocities corresponding to each subframe. The dots indicate the flow direction (entering the funnel from top) that was clearly known a priori from the experiment, and not recovered from the analysis. Note that some segments fall outside the funnel boundaries: this happens for those patches that partially intersect the funnel but have their centers outside the funnel. An overall rendering of the fluid flow was carried out by adopting the method for streamline visualization of vector fields proposed some years ago by Sparavigna et al. ${ }^{18}$ for showing liquid crystal defects. The method allows to obtain, starting from a 2D grid of the vector field [such as that of Fig. 7(c)], a continuous texture of oriented streamlines which depicts the overall features of the flow, and, at the same time, contains quantitative information about its orientation and strength. This is shown in Fig. 7(d), in which we used a scale of false colors similar to that of Fig. 7(c).

In order to check flux conservation, we measured the fluid flow passing through the various horizontal sections of the funnel. This check was carried out by recovering, from the data of Fig. 7(c), the vertical components of all the velocities, computing the flux in correspondence of each patch, and summing up the contributions of all the patches lying along each section. The calculation was worked out by taking into account Eq. (11) and by considering the effective cross sections of the patches located across the funnel border. The result is shown in Fig. 8 (open circles, left scale), in which the values of the flux are reported for the 16 horizontal sections of Fig. 7(c). As one can notice, with the exception of the last three points (top of the funnel), the flux is conserved remarkably well, with an average value of $0.235 \pm 0.003 \mathrm{~mm}^{3} / \mathrm{s}$ and a corresponding accuracy of the order of $1 \%$.

Finally, we also estimated the Reynolds number at the various horizontal sections. As known, for a noncircular duct, the Reynolds number can be defined empirically as, ${ }^{19}$

$$
\operatorname{Re}=\frac{\langle v\rangle d_{h} \rho}{\eta}
$$

where $\langle v\rangle$ is the average fluid velocity, $\rho$ is the fluid density, $\eta$ the fluid dynamical viscosity, and $d_{H}$ is the hydraulic diameter, ${ }^{19}$ of the duct defined as $d_{h}=4\left(A_{\mathrm{cs}} / P_{\mathrm{wt}}\right)$, being $A_{\mathrm{cs}}$ the duct cross section and $P_{\mathrm{wt}}$ the duct wetted perimeter. By using $\rho=1 \mathrm{~g} / \mathrm{cm}^{3}, \eta=0.01 \mathrm{~g} \mathrm{~cm}^{-1} \mathrm{~s}^{-1}$, the values for $\langle v\rangle$ recovered above, and considering that the cell has a square cross section of constant thickness $(1 \mathrm{~mm})$, we retrieved the values of $\operatorname{Re}$ at the various horizontal sections. The result, reported in Fig. 8 (solid squares, right scale), shows that the Reynolds number is almost constant along the funnel sections and is so small to ensure that the flux is fully laminar. 


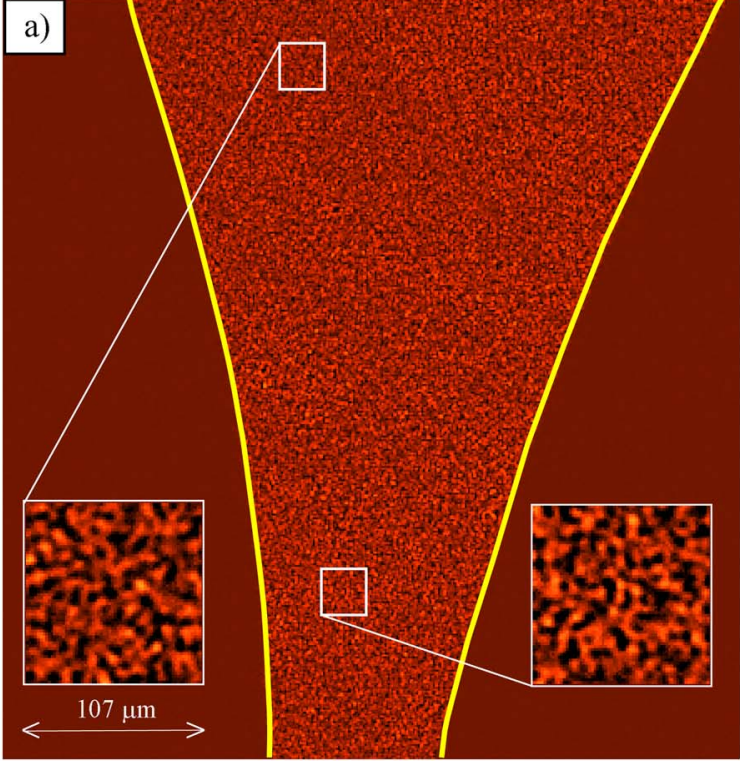

$1.72 \mathrm{~mm}$
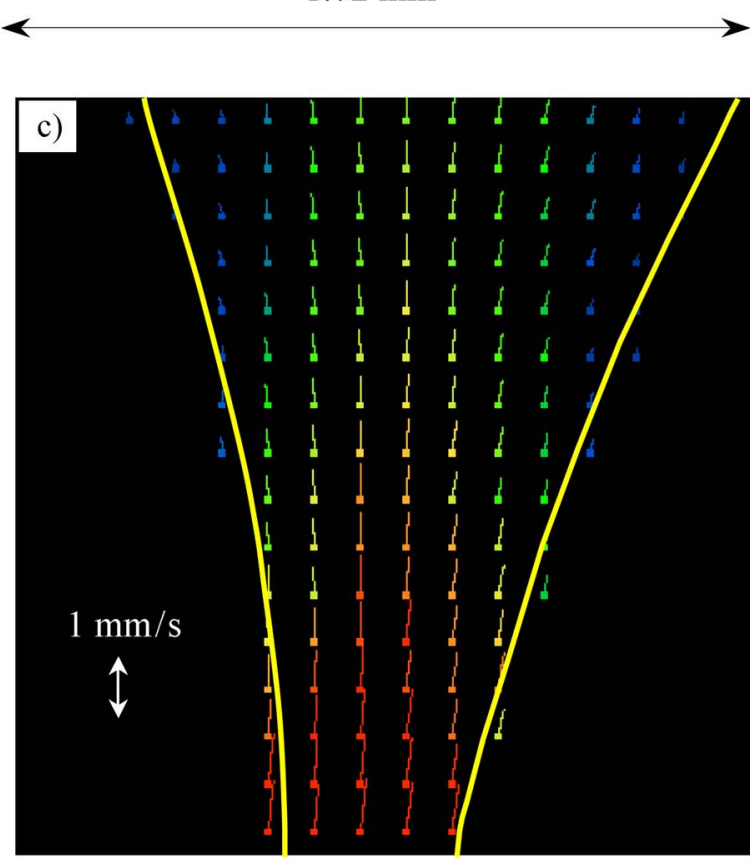

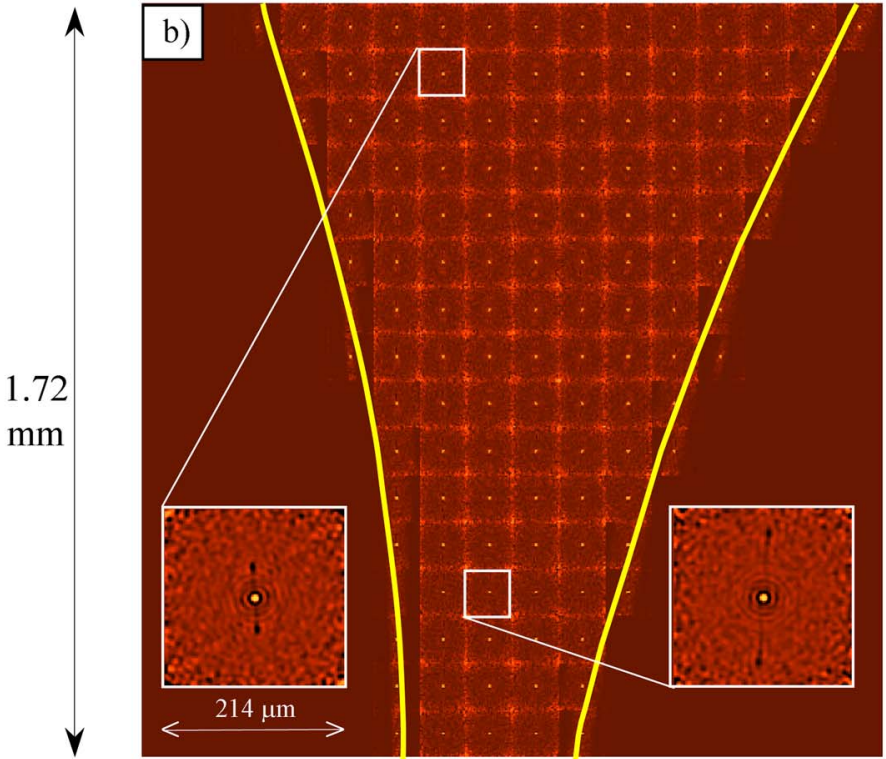

$1.72 \mathrm{~mm}$
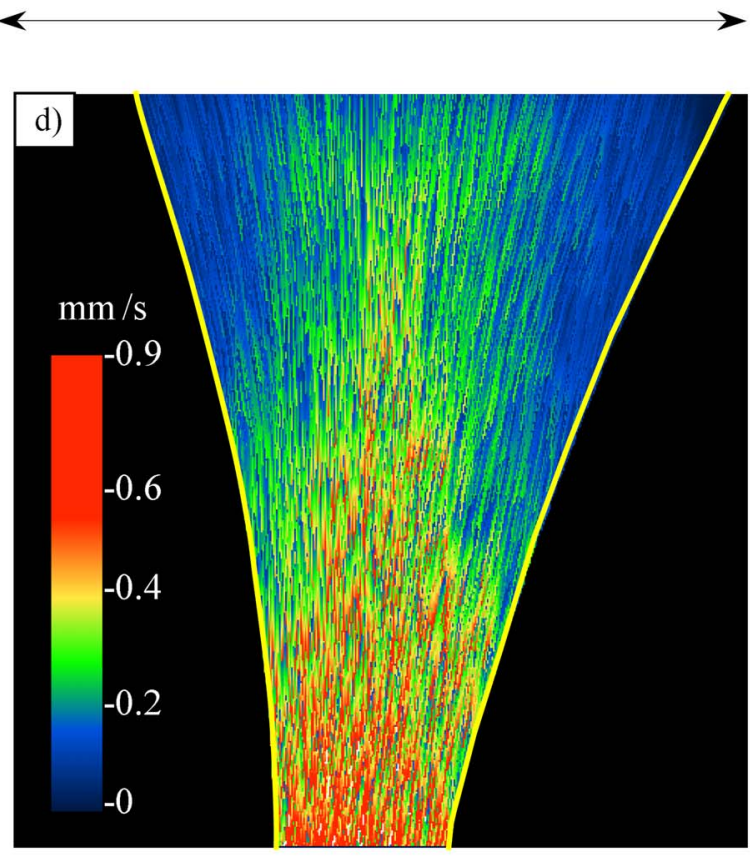

FIG. 7. (Color online) Images and analysis of the fluid flow through a funnel-shaped cell: (a) example of a differential heterodyne signal $\delta f(\mathbf{r}, t, \tau)$ for $\tau$ $=0.1 \mathrm{~s}$. (b) $2 \mathrm{D}$ mapping of the correlation function $g_{\tau}(\mathbf{x})$ obtained by dividing panel (a) into $16 \times 16$ subframes; the insets show the Poiseuille streams (see text). (c) Velocities mapping obtained from panel (b) by measuring length and directions of the Poiseuille streams. (d) Color rendering of the velocity streamline texture obtained from panel (c) as described in the text.

\section{CONCLUSIONS}

We have reviewed and discussed through numerical simulations and experiments the main features and performances of the technique heterodyne speckle velocimetry (HSV), which has been recently proposed as a new method for real-time velocimetry. HSV provides 2D mappings of the fluid velocities in the direction orthogonal to the optical axis and, at the same time, distributions of the fluid velocities over the entire sample thickness (longitudinal direction). HSV works well with small (submicron) particles and high particle concentration can be used as well. When compared with other classical velocimetry techniques such as PIV, LSV, LDV, or HPIV, our method appears to be somewhat less informative (for example, 2D instead of 3D), but offers several advantages related to its simplicity, sensitivity, and linearity. The last feature is probably the most important one, and it has been directly exploited in this work for the experimental characterization of a fluid moving under the conditions of Poiseuille flow.

In the first experiment we have shown that the velocity distribution expected in a liquid flowing between the two parallel vertical planes of a square cell with a constant cross 


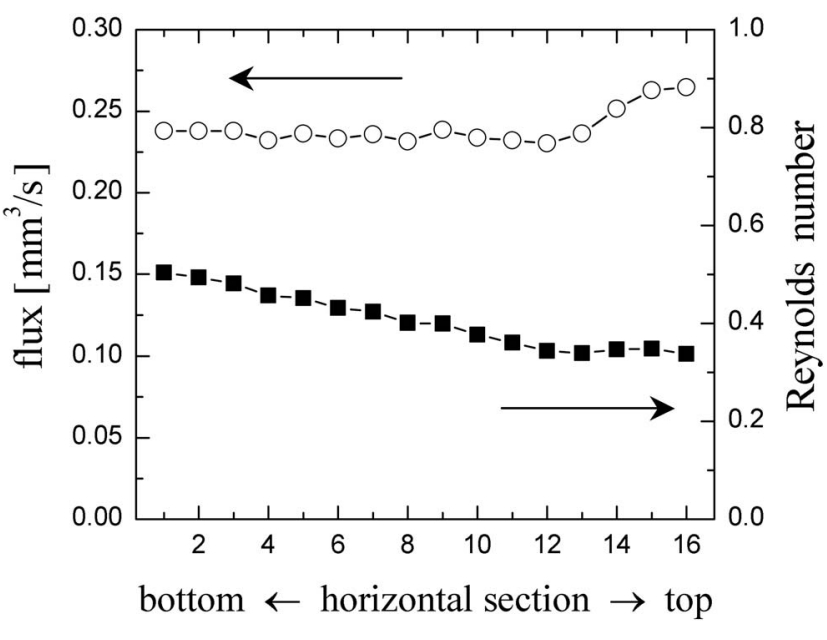

FIG. 8. Fluid flux (open circles, left scale) and Reynolds number (solid squares, right scale) as a function of the 16 horizontal sections of Fig. 7(c). The Reynolds number is defined by Eq. (12).

section can be easily recovered and matches rather accurately the Poiseuille theoretical expectations. In the second experiment the same investigation was carried out onto a funnelshaped cell, in which velocity gradients are present. The technique is able to reconstruct the 2D mapping of the velocity vectors, with the possibility of measuring the fluid flux within an accuracy of $1 \%$.

\section{ACKNOWLEDGMENTS}

We thank A. Sparavigna for having drawn to our attention the method for streamline visualization and for the analysis of the data reported in Fig. 7(d).

${ }^{1}$ R. J. Adrian, Annu. Rev. Fluid Mech. 23, 261 (1991).

${ }^{2}$ M. Raffel, C. Willert, and J. Kompenhans, Particle Image Velocimetry: A Practical Guide (Springer, Berlin, 1998).

${ }^{3}$ T. D. Dudderar, R. Meynart, and P. G. Simpkins, Opt. Lasers Eng. 9, 163 (1988).

${ }^{4}$ Y. Yeh and H. Z. Cummins, Appl. Phys. Lett. 4, 176 (1964).

${ }^{5}$ D. H. Barnhart, R. J. Adrian, and G. C. Papen, Appl. Opt. 33, 7159 (1994).

${ }^{6}$ Particle Image Velocimetry: Recent Improvements, edited by M. Stanislas,

J. Westerweel, and J. Kompenhans (Springer-Verlag, New York, 2004).

${ }^{7}$ H. E. Albrecht, M. Borys, N. Damaschke, and C. Tropea, Laser Doppler and Phase Doppler Measurement Technique (Springer, Berlin, 2003).

${ }^{8}$ H. Meng, G. Pan, Y. Pu, and S. H. Woodward, Meas. Sci. Technol. 15, 673 (2004).

${ }^{9}$ M. D. Alaimo, D. Magatti, F. Ferri, and M. A. C. Potenza, Appl. Phys. Lett. 88, 191101 (2006).

${ }^{10}$ M. Giglio, M. Carpineti, and A. Vailati, Phys. Rev. Lett. 85, 1416 (2000).

${ }^{11}$ D. Brogioli, A. Vailati, and M. Giglio, Appl. Phys. Lett. 81, 4109 (2002).

${ }^{12}$ F. Ferri, D. Magatti, D. Pescini, M. A. C. Potenza, and M. Giglio, Phys. Rev. E 70, 041405 (2004).

${ }^{13}$ J. W. Goodman, Speckle Phenomena in Optics (Roberts, Greenwood Village, CO, 2006).

${ }^{14}$ J. O. Sherer and L. P. Bernal, Appl. Opt. 36, 9309 (1997).

${ }^{15}$ J. W. Goodman, Statistical Optics (Wiley, New York, 1985), p. 174.

${ }^{16}$ L. D. Landau and E. M. Lifshitz, Fluid Mechanics (ButterworthHeinemann, Oxford, 2002).

${ }^{17} \mathrm{M}$. Kerker, The Scattering of Light and Other Electromagnetic Radiation (Academic, New York, 1969).

${ }^{18}$ A. Sparavigna, A. Sanna, B. Montrucchio, and A. Strigazzi, Liq. Cryst. 26, 1467 (1999).

${ }^{19}$ G. Gioia and F. A. Bombardelli, Phys. Rev. Lett. 88, 014501 (2001). 